\title{
Aceitação de metodologias de ensino à distância na área da saúde: Uma revisão integrativa
}

\author{
Study of the acceptance of e-learning methodologies in the health area: A \\ integrative review
}

\author{
Jéssica Louise Benelli \\ Mestre em Patologia, Biomédica na Empresa Brasileira de Serviços Hospitalares/ Hospital Universitário da Universidade Federal do Rio Grande, Rio \\ Grande do Sul. E-mail: jessicalb.jl@gmail.com \\ Lourdes da Silva Gil \\ Doutora em Ciências da Educação, Docente na Universidade Luterana do Brasil. E-mail: lourdes.gil@ globo.com
}

\begin{abstract}
Resumo: O presente estudo trata-se de uma revisão bibliográfica com o objetivo de avaliar a aceitação e inserção da metodologia EAD por estudantes e profissionais na área da Saúde, descrevendo metodologias realizadas, seus resultados e impacto na formação dos participantes. Para isso foi realizada busca em bases reconhecidas de artigos científicos que contenham as expressões: "EAD e saúde", "e-learning health", e "e-learning and health". Foram incluídos 43 artigos, dos quais 11 foram sobre metodologias nacionais, e os demais em diversos países de diferentes continentes. Na maioria das metodologias foi utilizado o ambiente virtual de aprendizagem, sendo o Moodle a plataforma mais citada, aplicada tanto a profissionais já formados, no contexto de formação continuada, quanto à alunos de graduação e pós-graduação, de modo complementar ao conhecimento presencial. Das iniciativas realizadas 90,5\% tiveram resultados positivos, algumas inclusive com resultados superiores aos de metodologias presenciais comparativas, sendo os resultados negativos associados à falta de engajamento dos tutores e professores ou a falta de dinamicidade da estratégia realizada. A presente revisão nos mostra que o ensino à distância tem sido utilizado para aprimorar, aumentar e consolidar conhecimentos inerentes à pratica dessas profissões de forma muito proveitosa, além disso, permite, na Saúde, a democratização do acesso aos recursos, sendo uma ferramenta também de Saúde Pública.
\end{abstract}

Palavras-chave: Ensino online; Formação continuada; Ambiente virtual

\begin{abstract}
The present study is a bibliographical review with the objective of evaluating the acceptance and insertion of the EAD methodology by students and professionals in the area of Health, describing methodologies, their results and their impact on the training of participants. For this purpose, a search was made on recognized bases of scientific articles containing the expressions "EAD and health", "e-learning health", and "e-learning and health". A total of 43 articles were included, of which 11 were on national methodologies, and the others in several countries on different continents. In most of the methodologies the virtual learning environment was used, being Moodle the most cited platform, applied to both professionals already trained in the context of continuing education, as well as undergraduate and postgraduate students, in a way complementary to face-to-face knowledge. Of the initiatives carried out, $90.5 \%$ had positive results, some of which were even better than comparative face-to-face methodologies, with negative results associated with the lack of engagement of tutors and teachers or the lack of dynamicity of the strategy. The present review shows that distance education has been used to improve, increase and consolidate knowledge inherent to the practice of these professions in a very useful way, besides, it allows, in Health, the democratization of access to resources, being a tool also of Public health.
\end{abstract}

Key words: Online education; Continuing education; Virtual environment 


\section{INTRODUÇÃO}

O Brasil é um país com enormes carências educacionais, neste contexto é imprescindível suprir as necessidades de educação com outras formas de aprimoramento do conhecimento (GONÇALVES et al., 2011). O modelo de educação na sociedade da informação, deve preconizar um ensino eficaz que considere o aluno como sujeito ativo e capaz de determinar o seu auto- aprendizado, além disso, favorecer a experimentação e o pensamento reflexivo (ABREU et al., 2003; CHAVES, 2007). É necessário a adoção de novas estratégias de educação que propiciem aprendizagem interativa e desenvolvimento de competências em pesquisa (GONÇALVES et al., 2011).

A Educação a Distância (EAD) vem sendo utilizada diante do processo intenso de globalização e criação de tecnologias inovadoras nas diversas áreas do conhecimento, tendo seu início na simples troca de emails e arquivos, o leque de possibilidades cresceu muito, como o uso de ambientes virtuais de aprendizagem (AVA) e simulações (PINTO; ROCHA, 2016). O avanço das tecnologias da informação e comunicação (TIC) impulsionaram este desenvolvimento (OLIVEIRA, 2007). A EAD é uma alternativa para a formação, pois facilita a disseminação das informações, diminui as barreiras geográficas e otimiza o tempo para o desenvolvimento das atividades (CAMACHO, 2009). Além disso a EAD surge como uma opção de solução da atual demanda de educação sendo um processo de aprendizagem eficaz (PICONEZ; FILASTRO, 2009).

O Ensino à Distância surgiu em 1904 no Brasil e hoje representa uma grande parcela dos alunos matriculados no Ensino Superior. No ano de 1904 têm-se o registro mais antigo de uma metodologia EAD no nosso país, através da divulgação no Jornal Brasil de cursos profissionalizantes (datilografia) por correspondência (BERTOLINI; DE MARCHI, 2010). Esse modelo que teve início na troca de cartas, evoluindo para o rádio e a televisão, cresceu muito, atendendo alunos da graduação e da pós-graduação e tendo na internet sua principal aliada, com o desenvolvimento de salas de aula e plataformas virtuais, como o MOODLE, Aulanet e WebCT (ABREU et al., 2003). Atualmente, a plataforma MOODLE é amplamente utilizada em metodologias EAD, além de outros recursos, como jogos online, simulações virtuais, grupos de discussão e outras formas de interação.

Dentro da área da saúde são muitas as dúvidas, preconceitos e questionamentos sobre a metodologia EAD, já que a Saúde é considerada, essencialmente, uma área prática. Esse crescimento constante dos recursos tecnológicos justifica a avaliação do emprego dos mesmos na área educacional e da sua efetividade e aceitação dentro da área da Saúde.

Por isso, o presente estudo tem como objetivo avaliar a aceitação de metodologias EAD na área da Saúde por parte dos alunos e profissionais participantes, baseando-se em relatos publicados em artigos científicos.

\section{MATERIAL E MÉTODOS}

O presente estudo consiste em uma revisão bibliográfica, realizada através da busca de artigos científicos indexados em bases de dados científicas. Foram utilizadas as bases de dados: Pubmed, Scielo e Lilacs. Para busca foram utilizadas as palavras chaves: "elearning health education", "e-learning and health" e "ead e saúde". Os artigos foram selecionados devendo conter a expressão e-learning ou EAD no título, escritos em português, inglês ou espanhol. Foram excluídos os artigos escritos em outras línguas, editoriais e cartas ao leitor.

A pesquisa no PUBMED trouxe 383 artigos dos quais 99 foram selecionados, no Scielo foram 13 escolhidos dentre 22 e no Lilacs 5 de 150, devido à grande sobreposição entre as bases de dados do PUBMED e do Lilacs. Nesta primeira etapa foram selecionados 117 artigos. A seguir foram lidos os resumos dos artigos.

A leitura dos resumos levou a exclusão de 71 artigos, sendo incluídos então 46. Dentre os artigos excluídos, 18 foram devido à ausência de descrição da metodologia EAD utilizada ou dos resultados, 23 por serem apenas artigos de revisão de conceitos, 4 por não estarem redigidos nas línguas incluídas nos critérios de inclusão (português, inglês e espanhol), 4 por aplicarem a metodologia EAD à pacientes e não à profissionais da saúde, 5 por não terem aplicado ainda a metodologia descrita, 12 por estarem duplicados no momento da pesquisa, 2 por não tratarem de temas relacionados à saúde e 1 por não apresentar metodologia EAD.

Após a leitura completa dos artigos, 3 foram excluídos por não apresentarem resultados das intervenções realizadas. Dessa forma a revisão bibliográfica contou com 43 artigos sobre a aceitação de metodologias EAD por estudantes e profissionais da saúde. Dos artigos lidos, foram extraídas informações sobre as metodologias utilizadas, público alvo, tema e os resultados obtidos.

\section{RESULTADOS E DISCUSSÃO}

\section{Estudos sobre o EAD na área da saúde no Brasil}

Diversos dos estudos selecionados discorrem sobre iniciativas desenvolvidas no Brasil, principalmente, no âmbito acadêmico em conjunto com hospitais universitários. Dos 43 estudos incluídos na revisão, 11 foram desenvolvidos por profissionais brasileiros em território nacional, principalmente, nas regiões Sul e Sudeste do País.

Jacominib et al. (2008), no estado de São Paulo, avaliaram a aceitação de um curso desenvolvido em ambiente virtual sobre Hepatite C, aplicado para enfermeiros graduados, como forma de formação continuada. $\mathrm{O}$ curso foi desenvolvido utilizando a plataforma Moodle e foi realizado com a disponibilização, durante o período de 5 semanas, de materiais de leitura e questionários. Dos 37 alunos convidados, 29 iniciaram o curso, e apenas 13 o concluíram. Dos concluintes, 50\% apresentou melhora do rendimento nas atividades avaliativas sobre o tema, e os demais mantiveram a mesma média, dos desistentes todos alegaram falta de tempo para realização das atividades. Entre os 
participantes cerca de $80 \%$ declaram-se muito satisfeitos com o curso.

Mezzari (2010), desenvolveu um estudo também baseado na plataforma Moodle para o ensino de Parasitologia à discentes do curso de Medicina de uma Universidade do Rio Grande do Sul. A plataforma virtual permitiu a aplicação da teoria da aprendizagem baseada em problemas (ABP) de forma mais intensa na disciplina, e 54,5\% dos alunos relatou preferir o método à distância do que o convencional, por este estimular a busca ativa pelo conhecimento.

Também na região sul, na cidade de Porto Alegre, Santos e Mercado (2010) relatam sua experiência com o Projeto POA_S@úde, desenvolvido como uma forma de telerradiologia, permitindo que usuários acessem imagens de exames radiológicos simultaneamente e a distância. O sistema Teleconsult permite o compartilhamento de exames de imagem e possibilita que especialistas compartilhem seus conhecimentos com residentes e médicos de regiões de menos acesso. O sistema foi aplicado com especialistas em medicina fetal e residentes de postos de atendimento da região da Restinga, em Porto Alegre, permitindo a capacitação e aprimoramento dos médicos e um melhor atendimento em regiões menos favorecidas.

Desenvolvido em 2009, 2010 e 2011, Silva et al. (2011) apresentaram suas impressões sobre curso na plataforma Moodle na área de Dermatologia aplicado a alunos de uma universidade de Salvador, Bahia. A carga horária dedicada à dermatologia é bastante restrita e o uso do ambiente virtual permitiu expandir e aprofundar o conhecimento dos alunos, apresentando grande aceitação dos alunos, 90\% consideraram o treinamento de grande importância. O estudo comparou um grupo presencial e um grupo EAD, e os resultados dos alunos EAD foram superiores aos do grupo controle.

Tobase et al. (2012) demonstram a importância do uso do EAD na formação continuada junto ao Sistema Único de Saúde (SUS), permitindo o aprimoramento de seus funcionários. $\mathrm{O}$ estudo apresenta o programa técnico de capacitação dos profissionais do SAMU em Suporte Básico de Vida (SBV), modalidade à distância, através da plataforma Moodle, permitindo a ampliação do treinamento em esfera nacional.

Camargo et al. (2014) compararam o uso de ferramentas EAD em graduandos e profissionais já graduados. A ferramenta utilizada foi a disponibilização de vídeo-aulas em DVD sobre tratamentos restaurativos com menos trauma, e os pesquisadores concluíram que os profissionais tiveram melhor aproveitamento do curso e maior incremento de conhecimento mensurado através de pré e pós-testes. Porém, longe de desacreditar o EAD na graduação, isso demonstra a importância da adequação do conteúdo e da estratégia pedagógica trabalhada ao público que se deseja alcançar, demonstrando que um tema tão específico exige conhecimentos prévios que os graduandos não possuíam, e que o curso era mais adequado como formação continuada.

Pinto e Rocha (2016) relatam os resultados da Rede de 16 Observatórios de Tecnologias de Informação e Comunicação em Serviços de Saúde - Rede OTICS-RIO - implementada pela Secretaria Municipal de Saúde do Rio de Janeiro no apoio à integração da atenção primária, vigilância e promoção da saúde, demonstrando mais uma vez a aplicação da metodologia EAD aos avanços educacionais e tecnológicos do SUS. A Rede OTICS-RIO consiste em espaço virtual, um blog, que incentiva a formação continuada dos profissionais e a disseminação do conhecimento, apresentando muitos resultados positivos em pouco tempo, entre 2011 e 2013, foram 1.195 reuniões/ workshops/ cursos/ oficinas/ seminários realizados com a presença de 44.894 pessoas circulando pelos observatórios.

Gangreia et al. (2016), no Estado de São Paulo, desenvolveram um ambiente virtual, do qual participaram 304 estudantes de medicina, ao qual chamaram de Rondas Virtuais, simulando casos e exigindo a tomada de decisões por parte dos estudantes, estimulando o estudo ativo, porém seguro, e aplicando a teoria da aprendizagem baseada em problemas (ABP), muito reconhecida no ensino da medicina e na área da Saúde. A avaliação do curso permitiu estabelecer uma correlação positiva entre o tempo de uso da plataforma e número de loggins semanais e o desempenho final na avaliação dos casos. O curso permitiu o desenvolvimento do pensamento rápido dos alunos e aumentou a confiança dos mesmos ao lidarem com pacientes reais.

Desde 2009, a Universidade Federal de Ciências da Saúde de Porto Alegre (UFCSPA) integra a rede UNASUS, que investe na formação continuada das equipes de saúde da família do Estado do Rio Grande do Sul, e a partir de 2012, estenderam esse projeto à região sul e nordeste (DAHMER et al., 2017). Dahmer et al. (2017) dissertam sobre os desafios da adequação do conteúdo a realidades tão diferentes dentro de um mesmo país. Os pesquisadores construíram um ambiente virtual baseado em casos clínicos escolhidos de acordo com as condições epidemiológicas de cada região, e demonstram a importância de, além do alcance do EAD, zelar pela qualidade e adequação do conteúdo proposto. Os relatos dos médicos participantes, permitiram concluir que mesmo uma Universidade do sul do país pode contribuir para o ensino em âmbito nacional.

Lau et al. (2017) avaliaram a percepção dos estudantes do internato de medicina de uma universidade de Minas Gerais, sobre duas atividades realizadas à distância, utilizando a plataforma Moodle. As atividades envolveram resolução de casos, construção de um webfólio e discussões em fóruns sobre Parasitologia Clínica, e $70 \%$ dos alunos declararam estarem mais preparados, após o curso, para as demandas reais do internato. A principal crítica dos estudantes foi em relação ao feedback dos professores, sendo que em $78 \%$ das postagens realizadas eles não obtiveram qualquer retorno do avaliador, e a média de dias para os feedbacks obtidos foi de 4,3 dias, sendo isso considerado um fator desestimulador para o uso da plataforma pelos discentes.

Oliveiras et al. (2017) também utilizaram a plataforma Moodle para conectar profissionais de 20 diferentes hospitais da região nordeste do país, com intuito de propiciar a formação continuada na área de Cardiologia Pediátrica, consistindo na disponibilização de vídeo-aulas e imagens. Dos inscritos no curso, 61\% concluíram o mesmo, sendo que o tempo máximo dedicado ao curso foi de 10 horas. Um sistema de avaliação pré e pós teste permitiu a comprovação do incremento de conhecimento 
dos profissionais, e $67 \%$ relataram satisfação total quanto ao curso. A tabela 1 sintetiza os dados apresentados, evidenciando as ferramentas utilizadas e o público abordado.

Tabela 1. Síntese demonstrativa do levantamento realizado relativo a Estudos Brasileiros sobre Metodologias EAD e sua aceitação na área da Saúde.

\begin{tabular}{|c|c|c|c|c|}
\hline Autor e Ano & Título & Ferramenta EAD & \multicolumn{2}{|c|}{ Público Alvo } \\
\hline Jacominib et al. (2008) & $\begin{array}{l}\text { Avaliação de um curso de } \\
\text { educação á distância sobre hepatite } \\
\text { C }\end{array}$ & Plataforma Moodle & $\begin{array}{l}\text { Profissionais } \\
\text { enfermagem }\end{array}$ & da \\
\hline Mezzari (2010) & $\begin{array}{l}\text { O Uso da Aprendizagem Baseada } \\
\text { em Problemas (ABP) como } \\
\text { Reforço ao Ensino Presencial } \\
\text { Utilizando o Ambiente de } \\
\text { Aprendizagem Moodle }\end{array}$ & Plataforma Moodle & Graduandos & \\
\hline Santos e Mercado (2010) & $\begin{array}{l}\text { Arquivamento e Comunicação de } \\
\text { Imagens Radiológicas na } \\
\text { Formação Médica Online }\end{array}$ & Telerradiologia & $\begin{array}{l}\text { Médicos } \\
\text { Residentes }\end{array}$ & $\mathrm{e}$ \\
\hline Silva et al. (2011) & $\begin{array}{l}\text { E-learning program for medical } \\
\text { students in dermatology }\end{array}$ & Plataforma Moodle & Graduandos & \\
\hline Tobase et al.(2012) & $\begin{array}{l}\text { Ensino à distância na educação } \\
\text { permanente em Urgência e } \\
\text { Emergência }\end{array}$ & Ambiente virtual & $\begin{array}{l}\text { Profissionais } \\
\text { SUS }\end{array}$ & do \\
\hline Camargo et al. (2014) & $\begin{array}{l}\text { Proposal of e-learning strategy to } \\
\text { teach Atraumatic Restorative } \\
\text { Treatment (ART) to undergraduate } \\
\text { and graduate students }\end{array}$ & Vídeos & $\begin{array}{l}\text { Graduandos } \\
\text { profissionais }\end{array}$ & $\mathrm{e}$ \\
\hline Pinto e Rocha (2016) & $\begin{array}{l}\text { Inovações na atenção Primária em } \\
\text { Saúde: o uso de ferramentas de } \\
\text { tecnologia de comunicação e } \\
\text { informação para apoio à gestão } \\
\text { local }\end{array}$ & $\mathrm{B} \log$ & $\begin{array}{l}\text { Profissionais } \\
\text { SUS }\end{array}$ & do \\
\hline Gangreia et al. (2016) & $\begin{array}{l}\text { Cognitive Load and Self- } \\
\text { Determination Theories Applied to } \\
\text { E-Learning:Impacton Students' } \\
\text { Participation and Academic } \\
\text { Performance }\end{array}$ & Ambiente virtual & Graduandos & \\
\hline Dahmer et al. (2017) & $\begin{array}{l}\text { Regionalização dos conteúdos de } \\
\text { um curso de especialização em } \\
\text { Saúde da Família, a distância: } \\
\text { experiência da Universidade } \\
\text { Aberta do Sistema Único de Saúde } \\
\text { (UNA-SUS/UFCSPA) em Porto } \\
\text { Alegre, Brasil }\end{array}$ & Ambiente Virtual & $\begin{array}{l}\text { Profissionais } \\
\text { SUS }\end{array}$ & do \\
\hline Lau et al. (2017) & $\begin{array}{l}\text { Implantação de Estratégias de } \\
\text { Ensino à Distância durante o } \\
\text { Internato: Desafios e Perspectivas }\end{array}$ & Ambiente virtual & Graduandos & \\
\hline Oliveira et al. (2017) & $\begin{array}{l}\text { Development and Assessment of } \\
\text { an E-learning Course on Pediatric } \\
\text { Cardiology Basics }\end{array}$ & Plataforma Moodle & $\begin{array}{l}\text { Profissionais } \\
\text { de hospitais }\end{array}$ & \\
\hline
\end{tabular}

\section{Estudos sobre o EAD na área da saúde no exterior}

Os estudos levantados apresentam experiências com EAD em todo o mundo durante uma década (20072017), com diferentes temas e públicos alvos, e todos com contribuições importantes para o desenvolvimento do EAD na Saúde, por isso a importância de reconhecermos essas abordagens e aprendermos com seus resultados.

Franson et al. (2007) avaliaram o desenvolvimento de mais de 2000 estudantes de Farmácia Clínica, durante os anos de 2003 a 2006, e assim como no estudo brasileiro de Gangreia et al. (2016), demonstraram que quanto maior o tempo empregado na ferramenta $\mathrm{EAD}$, em ambos os casos utilizando ambiente virtual (AVA), maior o rendimento do aluno. $\mathrm{O}$ estudo, desenvolvido em uma universidade dos Países Baixos, demonstrou que houve um aumento de $20 \%$ da nota final dos alunos que participaram da metodologia à distância. Estudo desenvolvido por Detroyer et al. (2016) na Bélgica, utilizando plataforma virtual, estruturada em 11 módulos de 5 a 15 minutos, tratando sobre pacientes psiquiátricos e formas de delírio, demonstrou que os profissionais que tiveram um aproveitamento bom ou excelente do curso, tiveram um tempo de uso até três vezes maior do que aqueles que tiveram um aproveitamento regular. 
Kulier et al. (2008) convidou 105 profissionais formados em Medicina, de diferentes países da Europa, a assistirem um curso em vídeo-aulas (de 15 a 20 minutos) disponibilizado em CD-ROM. Do total, 101 concluíram o curso, e todos apresentaram melhora na avaliação após a realização do curso, quando comparada com o pré-teste realizado.

Hadley et al. (2010), Países Baixos, desenvolveram um ensaio clínico randomizado, onde 91 estudantes foram alocados no grupo EAD e 79 no grupo de leitura e discussão presencial, com o objetivo de comparar a eficiência das duas modalidades de abordagens. $\mathrm{O}$ conteúdo se fundamentou na abordagem de aprendizagem baseada em problemas (ABP), trazendo casos clínicos, artigos e propiciando a discussão em fóruns. A análise comparativa não demonstrou diferenças nos resultados da metodologia presencial e EAD, sendo que a diferença do score da avaliação dos alunos foi de 0,1 pontos, demonstrando que a metodologia EAD é tão eficiente quanto a presencial, além de ser muito mais flexível.

Com metodologia e resultados semelhantes Abdelhai et al. (2012) relataram os resultados encontrados na avaliação de um curso sobre Saúde Reprodutiva aplicado a estudantes de uma escola médica do Egito, desenvolvido em quatro módulos com avaliações progressivas, na plataforma Moodle e comparado ao mesmo conteúdo administrado presencialmente. Os alunos foram avaliados antes e após a intervenção, e foi verificado que em ambas as metodologias houve incremento dos níveis de conhecimento. Da mesma forma não foram encontradas diferenças na eficácia das duas metodologias. Saúde reprodutiva, também foi o tema de um estudo desenvolvido na Suíça, por Abawi et al. (2016), cuja plataforma virtual atingiu alunos em 45 países diferentes em 6 continentes, sendo o maior percentual de estudantes Africanos, em dois anos onde mais de 300 alunos obtiveram certificação pelo curso.

Um estudo com metodologia muito semelhante foi realizado com alunos do curso de enfermagem de uma universidade do Irã, porém neste estudo, a metodologia EAD se mostrou superior em eficácia do que os grupos de leitura e discussão presenciais (MEHARDAD et al., 2011). Os estudantes relataram se sentirem mais motivados na metodologia $\mathrm{EAD}$, em um questionário sobre a sua experiência de estudo, cujas palavras mais citadas sobre a metodologia EAD foram "capacitação", "independência", "motivação" e "efetividade", mostrando grande adesão e aceitação dos alunos à metodologia. $\mathrm{O}$ estudo também demonstrou que os alunos da metodologia EAD perguntam e participam duas vezes mais do que os alunos da metodologia tradicional, mostrando a contribuição desse tipo de ensino para a formação de alunos ativos (MEHARDAD et al., 2011).

O'Leary (2012) desenvolveu em um hospital da Austrália, um curso de formação continuada à distância para profissionais médicos e da enfermagem recém graduados, sobre Suporte Básico e Avançado de Vida em Pediatria, com avaliação pré e pós realização da intervenção. Os efeitos do curso puderam ser constatados na prática clínica, onde se viu um incremento na detecção de arritmias e desfibrilações, contribuindo para o manejo adequado dos pacientes. Também na área de Pediatria,
Chang et al. (2014) desenvolveram curso semelhante à distância sobre emergências médicas, sendo detectado que os estudantes da metodologia EAD apresentaram resultados superiores aqueles em metodologia na modalidade presencial.

Mennon et al. (2012) desenvolveu uma atividade educacional com 14 terapeutas ocupacionais e fisioterapeutas canadenses sobre reabilitação de pacientes infartados. Os profissionais foram avaliados quanto à sua capacidade de buscar evidências científicas de qualidade em ferramentas específicas, como o StrokEngine, uma base de dados própria com os casos clínicos e suas repercussões. $\mathrm{O}$ estudo permitiu a reflexão sobre os casos e o treinamento dos participantes para a busca de evidências que contribuíssem para o seu trabalho.

O estudo de Nesterowickz et al. (2014), conduzido na Polônia, também se baseou em metodologias pré-existentes, sendo que profissionais formados em Farmácia foram convidados a participar de dois cursos EAD reconhecidos na área. Dos participantes, $92 \%$ já sabia da existência destes cursos online e $94 \%$ relatou estar muito satisfeito com a metodologia, sendo que houve um incremento de pontuação, na avaliação realizada após o término do curso, de $16 \%$, comparada ao pré-teste.

Houri et al. (2012) também desenvolveram uma atividade educacional para profissionais graduados em Medicina e outros cursos da Saúde, porém cerca de $70 \%$ dos participantes considerou a metodologia insatisfatória. Apesar dos resultados negativos, as considerações de Houri et al. (2012) são importantes no entendimento das necessidades dos alunos EAD. O curso foi desenvolvido como aulas em Power-Point com áudio, disponibilizadas em CD-ROM, os participantes (138) questionaram a adequação do conteúdo ao nível de ensino do público alvo, e a atualização das referências, que datavam de dois anos ou mais, bem como a total ausência de interação com um mediador ou tutor. Ebben et al. (2015) optaram pela mesma metodologia de disponibilização de material via CD-ROM, para equipe de paramédicos de um hospital dos Países Baixos, e tiveram um índice muito baixo de aderência, e falharam no seu objetivo que era fornecer uma atualização das novas diretrizes de cuidados com os pacientes.

Pode-se constatar com esse e outros relatos, que o sucesso de uma metodologia está associado à adequação e qualidade do conteúdo e com a interação dos alunos com o tutor. Metodologias online, como o uso de ambientes virtuais, permite uma atualização constante dos conteúdos, bem como a criação de fóruns e a interação se tornam maiores e mais interessantes, o que não existe em um curso disponibilizado de forma gravada e estática.

Haslerud et al. (2017) nos Países Baixos, desenvolveram um curso EAD sobre física médica para médicos que trabalham com diagnóstico com imagens, porém o curso se baseou apenas na disponibilização de leituras em PDF, sendo muito pouco aceito pelos alunos e criticado devido à complexidade do tema e a ausência de um tutor para gerir dúvidas e discussões.

Simmonsen et al. (2014) também encontraram desafios semelhantes, ao avaliar a efetividade de um curso de cálculos farmacêuticos para enfermeiras, viram que após o curso, $40 \%$ das participantes teve piora dos seus 
resultados, quando comparados ao pré-teste realizado, sendo que a principal dificuldade encontrada foi a ausência de feedback por parte dos tutores. Já Moczygemba et al. (2017) desenvolveram um curso EAD com a mesma temática, porém direcionado à farmacêuticos canadenses, e obtiveram $89 \%$ de aprovação dos participantes, sendo que $61 \%$ dos participantes afirmaram entender, após o curso, a teoria por trás de algumas decisões médicas, ressaltando a importância da correção das atividades realizadas.

Justamente a interação foi a aposta de um grupo de pesquisadores dos Países Baixos, realizada por Beurs et al. (2013) em um projeto denominado "Train-thetrainner". A metodologia consiste da formação de pequenos grupos interativos que mesclam profissionais de diferentes níveis, especialistas reconhecidos, tutores, trainners e estudantes, em um ambiente de discussões baseadas em vídeo-aulas, possibilitando uma troca muito rica de conhecimentos, permitindo o crescimento individual e coletivo e a dialogação de profissionais que ora se colocam como estudantes, ora como tutores. $\mathrm{O}$ tema das discussões foi o comportamento suicida, e após a intervenção, os participantes declararam considerarem-se mais aptos e confiantes para reconhecer os sintomas de comportamento suicida.

Boecker et al. (2013) utilizaram a diversão como forma de motivar seus alunos, um jogo desenvolvido em uma Universidade da Alemanha, simula a realização e interpretação de exames de urina, sendo que o grupo que estudou com o jogo foi comparado com um grupo de estudos baseados em leituras. Os estudantes, após igual período de treinamento e igual conteúdo nas metodologias nas quais foram alocados, foram submetidos à uma avaliação com questões objetivas, sendo que o grupo de estudos com o jogo virtual teve resultados $7,8 \%$ superiores aos do grupo de leitura, e quando questionados quanto a sua experiência educativa, os estudantes que utilizaram o jogo se demonstraram muito mais satisfeitos.

Taveira-Gomes et al. (2015), Portugal, seguiram a mesma linha de raciocínio proposta por Boecker et al. (2013), desenvolvendo um sistema de aprendizado baseado em "flash-cards", pequenos cartões informativos, sucintos e objetivos, com descrição de sintomas que os estudantes deveriam relacionar a suspeitas clínicas. Os cartões apresentavam informações resumidas e esquemas de cores de acordo com as diferentes doenças, facilitando a memorização de estudantes com facilidade visual, e foi considerado tão efetivo quanto à metodologia presencial, tradicionalmente utilizada. Garza et al. (2017) desenvolveram um website sobre cirurgia bariátrica, propondo um checklist para o desenvolvimento do procedimento e uma simulação virtual que permite o treino dos estudantes em procedimentos delicados, e foi demonstrado que os estudantes que utilizaram a metodologia tinham maior domínio da técnica do que os que não a utilizaram.

Mohamed et al. (2015) apresentaram os resultados de um grande investimento realizado no Sudão, na área de Telemedicina, demonstrando que a ideia apresentada por Santos e Mercado (2010) realizada em Porto Alegre (telerradiologia) tem um potencial muito maior a ser explorado ainda. No Sudão, 207 médicos de saúde da família espalhados pela região receberam laptops, com câmeras e microfones para gravação de imagem e som, e foram conectados à especialistas localizados no hospital de referência da capital. No período de um ano foram realizadas mais de 2000 consultas por especialidade, sendo a mais procurada a Pediatria, e foram atendidos 165.000 pacientes, levando um atendimento de referência a 84 pontos distantes da capital (MOHAMED et al., 2015).

A melhoria do acesso ao conhecimento, principalmente, em países em desenvolvimento, também foi a proposta de Barteit e Hophner (2015) dois pesquisadores alemães, que disponibilizaram computadores e acesso à internet para centros médicos na África, principalmente em Malawi, com o objetivo de capacitar estudantes de medicina da região. Para isso, os pesquisadores desenvolveram uma plataforma semelhante à de Gangreia et al. (2016), onde os alunos são guiados por rondas e pacientes virtuais, tomando as decisões conforme os casos são apresentados. Relatos dos participantes demonstraram que a plataforma foi crucial para a exercitação da tomada de decisões e do pensamento rápido, tão importante na lida com emergências, e que a principal dificuldade foi o acesso à tecnologia, sendo que a melhora proporcionada pelo projeto, trouxe grande contribuição para formação dos estudantes.

A melhoria do discernimento e avaliação dos casos também foi o grande diferencial da "Virtual School of TUMS", escola virtual de simulação de uma universidade de Teerã, no Irã, apresentada por Gharib et al. (2016) que possibilitou o aumento de horas de treino dos alunos, sem comprometer a segurança dos pacientes, da mesma forma que a plataforma virtual desenvolvida na Alemanha, por Gaupp et al. (2016) e aplicada a 321 estudantes de medicina, treinados para melhorar as condições de segurança do paciente.

$\mathrm{O}$ ensino EAD não trata apenas de questões práticas ou técnicas, como relatado anteriormente, mas há também preocupação com a segurança do paciente, o desenvolvimento do discernimento e confiança dos estudos e da humanização do atendimento. Nesse sentido, Seeland et al. (2016) desenvolveram, através da plataforma Moodle, o curso eGender, que trata sobre questões de gênero e sexualidade, que já foi utilizada por mais de 3000 participantes, entre estudantes e profissionais, permitindo um meio de interação muito rico, onde $45 \%$ concordaram que o curso melhorou seu entendimento e manejo dos e com os pacientes, e $55 \%$ concordaram fortemente.

Bos-Bonnie et al. (2017), também trabalharam a sensibilidade dos estudantes e profissionais na abordagem do histórico sexual e doenças sexualmente transmissíveis (DSTs) com os pacientes, através de uma plataforma de educação continuada, sendo que $42,5 \%$ relataram maior segurança ao abordar o histórico sexual com os pacientes e $34,5 \%$ disseram já ter implementado as mudanças comportamentais na triagem aprendidas no curso.

Yao et al. (2016) desenvolveram em ambiente virtual, com vídeos, leituras e imagens, um curso para diagnóstico precoce de lesões gástricas indicativas de câncer de estômago, e avaliaram os seus alunos comparando os mesmos com um grupo de estudo presencial. Na avaliação prévia ao curso, os dois grupos tiveram aproveitamento de $52 \%$ do teste, porém após o 
curso, os estudantes EAD tiveram um incremento de dez pontos, com aproveitamento de $62 \%$, enquanto que o grupo presencial não teve melhora da pontuação.

$\mathrm{Xu}$ et al. (2016) desenvolveram uma plataforma de discussão de evidências médicas disponibilizada em todo território Chinês, e viram que os participantes que no início do curso tinham média de avaliação sobre primeiros socorros e emergências de $55 \%$, após o curso tiveram um aproveitamento de $85 \%$, demonstrando claramente a efetividade do ensino EAD. Satisfação semelhante, foi encontrada por Hashemikamangar et al. (2016), de uma universidade de Teerã, no Irã, que desenvolveram um curso com profissionais formados na área de Odontologia.

Degerfält et al. (2017) apresentam o estudo de maior duração dos analisados, avaliando um projeto de formação continuada em Oncologia, desenvolvido na Dinamarca, em ambiente virtual, com dados já avaliados e obtidos desde 2005 a 2014, sendo que $68 \%$ dos usuários considerou (a iniciativa excelente e, também, 45\% considerou que o programa apresentou um nível muito alto de evidências científicas, contribuindo para o conhecimento nacional sobre o tema.

Mistraletti et al. (2017), na Itália, desenvolveram um website com cursos de atualização das diretrizes adotadas internacionalmente no manejo de pacientes com dor, alucinações e delírios, sendo que em 9 meses, 314 médicos e enfermeiros participaram da iniciativa e mais de $30 \%$ chegou a concluir dois ou mais cursos de formação.

\section{Análise dos estudos incluídos}

A área da Saúde é entendida como um ramo muito prático do conhecimento, aprendido com “ a mão na massa", frente-a-frente com o paciente, mas devemos considerar formas de melhor preparar os alunos e profissionais da área para o exercício de suas atividades.

Ao conversar com profissionais da área da Saúde, colegas de profissão, observa-se uma visão muito mais limitada do que a realidade do EAD na área da Saúde, esse "pré-conceito" se deve à muitos medos, dúvidas e inseguranças, que são gerados pelo desconhecimento e despreparo para o uso de metodologias à distância. No entanto, o número de publicações sobre o tema tem crescido significativamente, bem como, a riqueza e complexidade das iniciativas desenvolvidas, com relatos muito interessantes espalhados por todo o mundo.

A presente revisão bibliográfica teve como objetivo construir um retrato da metodologia de Ensino à Distância (EAD) na área da Saúde, vendo sua aceitação e inserção neste meio. O primeiro ponto que foi observado é o grande número de material disponível em revistas indexadas e reconhecidas, também chama atenção a parcela desses artigos que são produzidos por brasileiros, mostrando o quanto que nosso país tem investido nessa metodologia.

Todos os artigos estão compreendidos no período de uma década (2007-2017), o que demonstra o crescente interesse pelo EAD, e o aumento das publicações sobre o tema. As iniciativas têm sido aprimoradas demonstrando melhores resultados. Dos artigos, 13 foram publicados em revistas que tratam exclusivamente de Educação e $30 \mathrm{em}$ revistas sobre ciência e medicina, demonstrando um crescente interesse e reconhecimento pela educação e formação dos profissionais da área da Saúde. A publicação em grandes revistas internacionais demonstra que o ensino EAD tem ganho grande destaque.

Do total de estudos incluídos nesta revisão (43) 11 são sobre iniciativas brasileiras, e os demais 32 abrangem todo o mundo. Das 11 intervenções realizadas no Brasil, 6 foram com profissionais e 5 com estudantes. Inclusive, podemos constatar que o EAD tem sido utilizado, de forma eficaz, como educação continuada junto aos profissionais do SUS, como podemos observar nas metodologias descritas por Tobase et al. (2012), Dahmer et al. (2017) e Pinto e Rocha (2016). O Ensino à Distância se torna uma extensão do conhecimento prática e fácil de ser levada a regiões com menos recursos, como o projeto em áreas carentes de Porto Alegre, desenvolvido por Santos e Mercado (2010), em uma iniciativa de Telemedicina.

Entre os demais relatos encontram-se metodologias desenvolvidas nos países: África, Alemanha, Austrália, Bélgica, Canadá, China, Dinamarca, Egito, Estados Unidos da América, Irã, Itália, Japão, Países Baixos, Polônia, Portugal, Sudão e Suíça. Dessa forma podemos ver que o Brasil tem contribuído de forma importante com metodologias EAD e publicação dos seus resultados, bem como podemos afirmar que a metodologia do ensino à distância se espalhou por todo o mundo, encurtando distâncias e facilitando o acesso. A questão de facilitação do acesso foi citada fortemente por Santos e Mercado (2010), em Porto Alegre, Mohamed et al. (2015), no Sudão, Barteit et al. (2015), que desenvolveram atividades na África, e utilizaram as ferramentas EAD para levar conhecimento e a medicina a áreas menos privilegiadas e com menos recursos.

As intervenções foram realizadas em cursos de pós-graduação, graduação, em ambiente acadêmico e hospitalar, demonstrando a difusão da prática, 25 das intervenções teve por objetivo o público profissional, já formado (sendo citadas as profissões do médico, enfermeiro, fisioterapeuta e farmacêuticos) e 18 foram voltadas para alunos ainda na graduação, especialmente, de enfermagem e de medicina.

As intervenções utilizadas foram bastante diversificadas, de forma que os recursos do EAD e os recursos tecnológicos foram importantes nesse sentido. De longe, o meio mais utilizado foi o desenvolvimento de ambientes virtuais de aprendizagem (AVA), utilizado em 30 das intervenções, sendo que a plataforma Moodle foi citada em 20 destas. Outras ferramentas utilizadas foram: áudio e leitura (4 citações); website (3 citações); telemedicina (2 citações); ferramentas de busca (1 citação); grupo interativo (1 citação); jogos (1 citação); e blogs (1 citação). Os temas abordados são os mais diversos, incluindo áreas de Reprodução Humana, Infectologia, Sexualidade, Oncologia, Cardiologia, Pediatria, Cirurgia Geral, Diagnóstico por Imagem, Cálculos farmacêuticos, entre outros.

O ambiente virtual de aprendizagem é uma ferramenta muito popular no EAD pela sua riqueza em recursos, pois dentro do mesmo pode englobar vários outros recursos. A maioria dos cursos citados é organizado em módulos que possuem questionários pré-teste e pós teste, aulas em vídeo, slides ou textos disponibilizados para leitura. O AVA permite o envio de arquivos, 
resolução de atividades, enquetes, avaliações, construções de wikipédias, discussão em fóruns, envio de links, avaliação e feedback do professor, além de permitir o controle dos loggins dos alunos e do tempo de acesso, medidas que são correlacionadas com os resultados obtidos no curso e utilizadas como medidas de adesão. $\mathrm{O}$ uso do Moodle é amplamente difundido, por ser essa uma ferramenta muito útil devido à sua inserção com os recursos tecnológicos e o grande leque de possibilidades.

A maioria dos resultados das intervenções foi positivo, contribuindo para o estabelecimento da metodologia à distância como uma prática eficaz e de qualidade. Dos resultados apresentados 39 foram positivos $(90,5 \%)$, enquanto que 4 estudos relataram resultados negativos $(9,5 \%)$. Como pontos positivos citados estão o incremento de conhecimento, aumento de pontuação pósteste, participação, acesso à recursos, motivação dos alunos, certificação dos mesmos e a formação de alunos mais ativos, que buscam seu próprio conhecimento, como citado por Mezzari (2011). Inclusive Mehardad et al. (2011) e Chang et al. (2014) ao compararem metodologias EAD e presenciais na saúde, destacaram que as metodologias à distância foram superiores em efetividade às presenciais. Este conhecimento também se torna mais seguro, preparando o aluno em um ambiente controlado, Gharib et al. (2016) e Gaupp et al. (2017) desenvolveram simulações do ambiente hospitalar, formando o senso crítico e o poder de decisão desses alunos sem colocar em risco à segurança dos pacientes, e aumento seu preparo ao lidarem com pessoas reais.

Claro que nesse trajeto também existem desafios, e o mais recorrente nos resultados apresentados é a dificuldade de aderência, ou seja, muitos alunos iniciam o curso, mas não o concluem, como relatado por Jacominib et al. (2008) e Ebben et al. (2015). Um fator determinante para a aderência dos alunos ao curso é o engajamento dos professores. Todas as iniciativas que apresentaram resultados negativos $(9,5 \%$ dos casos) relataram baixo ou nenhum nível de interação com tutores, professores ou entre os próprios alunos, o que, segundo Houri et al. (2012) e Lau et al. (2017) resultou em grande insatisfação dos usuários. Nessas iniciativas também, houve reclamações sobre os professores que não retornavam as avaliações, mostrando que o aluno dá grande valor ao feedback do professor para orientar seus estudos. Isso demonstra que o engajamento e o preparo dos professores para trabalhar com metodologias à distância é determinante no sucesso ou falha das mesmas.

Todas as iniciativas foram utilizadas de maneira complementar ao ensino presencial, ou seja, simultaneamente à graduação, ou como formação continuada, mostrando que o uso exclusivo do EAD na área da Saúde é muito difícil e pouco aceito. As iniciativas variam desde pequenos ensaios com pequenos grupos, até grandes intervenções em nível nacional, um dos trabalhos que merece destaque é o de Mohamed et al. (2015), realizado no Sudão, que a partir do uso da Telemedicina, permitiu o acesso de especialistas em muitas outras localidades com escassez de recursos. Isso mostra que além de um mecanismo de educação, o EAD quando empregado na Saúde é um mecanismo humanitário, com potencial para melhorar a qualidade do atendimento médico dos pacientes, incluindo a agilidade e maior eficácia em relação a tempo de atuação

Apesar da grande maioria dos resultados serem positivos, temos que considerar que estes dados podem estar superestimados, devido a limitações reconhecidas do estudo. A presente revisão abrangeu apenas artigos publicados, e é conhecido o viés de publicação, que determina que artigos com resultados positivos tendem a ter mais chance de serem aceitos do que aqueles com resultados negativos. Apesar, dessa ânsia natural do ser humano em demonstrar e valorizar o acerto, é inquestionável o valor da aprendizagem associado ao erro, de forma que esses artigos com resultados negativos são tão importantes para nossa análise quanto os positivos.

$\mathrm{O}$ ensino à distância na saúde tem sido utilizado para aprimorar, aumentar e consolidar conhecimentos inerentes à prática dessas profissões, de forma muito proveitosa. Além disso, quando pensamos em Saúde, e se extrapolarmos o contexto da formação acadêmica, nos direcionando ao tripé ensino-pesquisa-extensão, o EAD na Saúde se torna uma força humanitária. Não é exagero dizer isso, se considerarmos que com a Telemedicina é possível levar à 84 localidades diferentes no Sudão, especialistas, que de outro modo estariam disponíveis apenas na capital, e que através do uso dessa tecnologia atenderam 165.000 pacientes em um ano (MOHAMED et al., 2015). Sabemos da carência de recursos e de profissionais da Saúde em todo o mundo, e o EAD pode contribuir na democratização desses recursos tão importantes à manutenção da vida.

\section{CONCLUSÕES}

As metodologias para a Educação à distância têm sido bem aceitas e bastante efetivas, tanto quando aplicadas aos estudantes quanto aos profissionais da área da Saúde, apresentando grande importância na formação continuada e capacitação dos profissionais da Saúde. O uso dessas metodologias tem alta satisfação justificada por características relacionada ao conhecimento, acessibilidade e segurança dos métodos utilizados.

\section{REFERÊNCIAS}

ABAWI，K.; CHANDRA-MOULI， V.; TOSKIN, I.; FESTIN, M. P.; GERTISER, L.; IDRIS, R.; HAMAMY, H.; ALI, M.; BONVENTURE, A. M.; TEMMERMAN, M.; CAMPANA, A. E-learning for research capacity strengthening in sexual and reproductive health: the experience of the Geneva Foundation for medical education and research and the department of reproductive health and research, World Health Organization. Human Resources for Health, v. 14, n. 76, 2016.

ABDELHAI, R.; YASSIN, S.; AHMAD, M. F.; FORS, U. G. H. An e-learning reproductive health module to support improved student learning and interaction: a prospective interventional study at a medical school in Egypt. BMC Medical Education, v.12, n. 11, 2012.

ABREU, A. F.; GONÇALVES, C. M.; PAGNOZZI L. Tecnologia da informação e educação corporativa: contribuições e desafios da modalidade de ensino- 
aprendizagem à distância no desenvolvimento de pessoas. Rev PEC, v.3, n.1, p. 55-8, 2003.

BARTEIT, S.; HOEPFFNER, P.; KARAMAGI, A.; MUNTHALI, C.; THEURER, A.; NEUHANN, F. Selfdirected e-learning at atertiary hospital in Malawi- A qualitative Evaluation and Lessons learn. GMS Zeitschriftfür Medizinische Ausbildung, v.32, n.1, 2015.

BERTOLIN J. C. G.; DE MARCHI A. C. B. Instrumentos para avaliar disciplinas da modalidade semipresencial: uma proposta baseada em sistemas de indicadores. Avaliação, v. 15, n. 3, p. 131-146, 2010.

BEURS, D. P.; GROOT, M. H.; KEIJSER, J.; VERWEY, B.; MOKKENSTORM, J.; TWISK, J. T. R.; VAN DUJIN, E.; VAN HEMERT, A. M.; VERLINDE, L.; VAN LUJIN, B.; VINK, J.; KHERKHOF, A. J. Improving the application of a practice guideline for the assessment and treatent suicidal behavior by training the full staff of physichiatric departaments via an e-learning supported Train-the-Trainner Program: study protocol for the randomised controlled trial. Trials, v.14, n. 9, 2013.

BOEKER, M.; ANDEL, P.; VACH, W.; FRANKENSCHMIDT, A. Game-based e-learning is more effective than a conventional instructional method: a randomized controlled trial with third-year medical students. Plos ONE, v.8, n.12, 2013.

BOS-BONNIE, L. H. A.; JAN, E. A. M.; PAS, E.; KIJSER, M.; DIJK, N. Effectiveness of an individual, online e-learning program about sexually transmitted infections: a prospective cohort study. BMC Family Practice, v.18, n.57, 2017.

CAMACHO A. C. L. F. Análise das publicações nacionais sobre educação a distância na enfermagem. Rev Bras Enferm, v.62, n.4, p.588-93, 2009.

CAMARGO, L. B.; RAGGIO, D. P.; BONACINA, C. F.; WEN, C. L; MENDES, F. M.; BONECKER, M. J. S.; HADDAD, A. E. Proposal of e-learning strategy to teach Atraumatic Restorative Treatment (ART) to undergraduate and graduate students. BMC Research Notes, v.7, 2014.

CHANG, T. P.; PHAM, P. K.; SOBOLEWSKI, B.; DOUGHTY, C. B.; JAMAL, N.; KWAN, K. Y.; LITTLE, K.; BRENKERT, T. E.; MATHISON, D.J. Pediatric emergency medicine asynchronous e-learning: a multicenter randomized controlled solomon four-group study. Academic Emergency Medicine, v.21, n.8, p.912-9, 2014.

CHAVES E. Tecnologias na educação: conceitos básicos [texto na Internet]. Disponível em: http://edu tec.net/Tecnologia $\% 20 \mathrm{e} \% 20$ Educacao/edconc.htm.

Acesso em 01 de nov de 2017.

DAHMER, A.; PORTELLA, F. F.; TUBELO, R. A.; MATTOS, L. B.; GOMES, M. Q.; COSTA, M. R. Regionalização dos conteúdos de um curso de especialização em Saúde da Família, a distância: experiência da Universidade Aberta do Sistema Único de Saúde (UNA-SUS/UFCSPA) em Porto Alegre, Brasil. Comunicação, Saúde e Educação, v. 21, n.61, p.449-63, 2017.

DEGERFÄLT, J.; SJÖSTEDT， S.; FRANSSON， P.; KJELLÉN, E.; WERNER, M. U. E-learning programs in oncology: a nation wide experience from 2005 to 2014. BMC Res Notes, v.10, n.39, 2017.

DETROYER, E.; DOBBELS, F.; DEBONNAIRE, D.; IRVING, K.; TEODORCZUK, A.; FICK, D.; JOOSTEN, E.; MILSEN, K. The effect of an interactive delirium elearning tool on healthcare workers' delirium recognition, knowledge and strain in caring for delirious patients: a pilot pre-test/post-test study. BMC Medical Education, v.16, n.17, 2016.

EBBEN, R. H. A; GRUNSVEN, P. M.; MOORS, M. L.; ALDENHVEN, P.; VAAN, J.; VAN HOUT, R.; VAN ACTERBERG, G.; VLOET, L. C. A tailored e-learning improve program to improve handover in the chain of emergency care: a pre-test post-test study. Scandinavian Journal of Trauma, Resuscitation and Emergency Medicine, v.23, n.33, 2015.

FRANSON, K. L.; DUBOIS, E. A.; KAM, M. L.; COHEN, A. F. Measuring learning from the TRC pharmacology E-Learning program. Britinish Journal Clinical Pharmacoly, v.66, n.1, p.135-41, 2007.

GARZA，J.; KOWALEWSKI， K.; FRIEDRICH， M.; SCHMIDT, M. W.; BRUCKNER, T.; KENNGOTT, H. G.; FISCHER, L.; MÜLLER-STICH, B. P.; NICKEL, F. Does rating the operation videos with a checklist score improve the effect of e-learning for bariatric surgical training? Study protocol for a randomized controlled trial. Trials, v.18, n.134, 2017

GAUPP, R.; KÖRNER, M.; FABRY, G. Effects of a casebased interactive elearning course on knowledge and attitudes about patient safety: a quasiexperimental study with third-year medical students. BMC Medical Education, v.16, 2016.

GHARIB, M.; ZOLFAGHAR, I. M.; MOJTAHEDZADEH, R.; MOHAMMADI, A.; GHARIB, A. Promotion of critical thinking in e-learning: a qualitative study on the experiences of instructors and students. Advances in Medical Education and Practice, v.7, p.271-9, 2016.

GONÇALVES, T. S.; CRENITTE, P. A. P. Development of a CD-ROM on wrien language for the connuing educaon of elementar school teachers. J Appl Oral Sci v. 19, n.6, p.560-6, 2011.

GRANGEIA, T. A. G.; JORGE, B.; FRANCI, D.; SANTOS, T. M.; SETUBAL, M. A. V.; SCHWELLER, M.; DE CARVALHO-FILHO, M. A. Cognitive load and self-determination theories applied to e-learning: impacton 
students' participation and academic performance. PLOS ONE, v.11, n.3, 2016.

HADLEY, J.; KULIER, R.; ZAMORA, J.; COPPUS, S.; WEINBRENNER, S.; MEYERROSE, B.; DECSI, T.; HORVATH, A. R.; NAGY, E.; EMPARANZA, J. I.; ARVANTIS, T. N.; BURLS, A.; CABELLO, J. B.; KACZOR, M.; ZANHEI, G.; PIERER, K.; KUNZ, R.; WILKIE, V.; WALL, D.; MOL, B. W.; KHAN, K. S. Effectiveness of an e-learning course in evidence-based medicine for foundation (internship) training. J R Soc Med, v. 103, n.7, p.288-94, 2010.

HASHEMIKAMANGAR, S. S.; YAZDANPANAH, F.; MIRZAII, M.; YAZDANI, R.; KARAZIFARD, M. J.; YASINI, E. Efficacy of e-learning via the website of Tehran University of medical sciences for diagnosing tooth discolorations and treatment planning by senior dental students. Acta Medica Iranica, v.54, n.8, 2016.

HASLERUD, T.; TULIPAN, A. J.; GRAY JR., R. M.; BIERMANN, M. E-learning for medical imaging specialists: introducing blended learning in a nuclear medicine specialist course. Acta Radiologica Open, v.6, n.7, p.1-5, 2017.

HOURI, D.; WATANABE, T.; HAYASHI, K.; KUROZAWA, Y. Evaluation of an e-learning distance education system in the graduate school of medical sciences of Tottori University. Yonago Acta medica v.55, p.69-73, 2012.

JACOMINIB, R. A; PIAIC, T. H.; FIGUEIREDO, R. M. Avaliação de um curso de educação á distância sobre hepatite C. Invest Educ Enferm. v.26, n.2, p.98-104, 2008.

KULIER, R.; HADLEY, J.; WEINBRENNER, S.; MEYERROSE, B.; DECSI, T.; HORVATH, A. R.; NAGY, E.; EMPARANZA, J. I.; COPPUS, S. F.; ARVANITIS, T. N.; BURLS, A.; CABELLO, J. B.; KACZOR, M.; ZANREI, G.; PIERER, K.; KUNZ, R.; MOL, B. W.; KHAN, K. S. Harmonising evidence-based medicine teaching: a study of the outcomes of e-learning in five Europe countries. BMC Medical Education, v.8, n. 7, 2008.

LAU, F. A.; MENDES, V. F.; VALDES, A. A. V.; BOLLELAI, R.; TEIXEIRA, L. A. S. Implantação de Estratégias de Ensino à Distância durante o Internato: Desafios e Perspectivas. Revista Brasileira de educação Médica, v.41, n.2, p.269-77, 2017.

MISTRALETTI, G.; UMBRELLO, M.; ANANIA, E.; ANDRIGHUI, E.; DI CARLO, A .; MARTINETTI, F.; BARELLO, S.; SABBATINI, G.; FORMENTI, P.; MARAFFI, T.; MARAZZO, F.; PALO, A.; BELLANI, G.; RUSSO, R.; FRANCESCONI, S.; VALDOMBRINI, F.; CIGADA, M.; RICCARDI, F.; MOJA, E. A., IAPICHINO, G.; SEDALCU INVESTIGATORS. Neurological assessment with validated tools in general icU: multicenter, randomized, before and after, pragmatic study to evaluate the effectiveness of an e-learning platform for continuous medical education. Minerva anestesiologica, v.83, n.2, p.145-54, 2017.

MEHRDAD, N.; ZOLFAGHARI, M.; BAHRANI, N.; EYBPOOSH, S. Learning outcomes in two different teaching approach in nursing education in Iran: e-learning versus lecture. Acta Medica Iranica, v.49, n.5, p.296-9, 2011.

MENON, A.; KORNER-BITENSKY, N.; CHIGNELL, M.; STRAUS, S. Usability testing of two e-learning resources: methods to maximize potential for clinical use. J Rehabil Med, v.44, p.338-45, 2012.

MEZZARI, A. O uso da aprendizagem baseada em problemas (ABP) como reforço ao ensino presencial utilizando o ambiente de aprendizagem Moodle. Revista Brasileira de Educação Médica, v.35, n.1, p.114-21, 2010.

MOCZYGEMBA, L. R.; PIERCE, A . L.; DANG, A.; EMBERLEY, P.; CZAR, M. J.; MATZKE, G. R. The ADAPT online education program: A tool for practicing pharmacists delivering patient-centered care. Journal of the american pharmacists Association, v.57, n.5, p.601-7, 2017.

MOHAMED, K. G.; HUNSKAAR, S.; ABDELRAHMAN, S. H.; MALIK, E. M. Telemedicine and e-learning in a primary care setting in Sudan: the experience of the gezira family medicine project. International Journal of Family Medicine, v.2015, 2015.

NESTEROWICKZ, K.; LIBROWSKI, T.; EDELBRING, S. Validanting e-learning in continuing pharmacy education: user acceptance and knowledge change. BMC Medical Education, v.14, n.33, 2014.

O'LEARY, F. M. Paediatric resuscitation training: Is elearning the answer? A before and after pilot study. Journal of Paediatrics and Child Health, v.48, p.529-33, 2012.

OLIVEIRA M. A. N. Educação a distância como estratégia para a educação permanente em saúde: possibilidades e desafios. Rev Bras Enferm, v..60,n.5, p.585-9, 2007.

OLIVEIRA, A. C.; MATTOS, S.; COIMBRA, M. Development and assessment of an e-learning course on pediatric cardiology basics. JMIR Med Educ, v.3, n.1, 2017.

PICONEZ S. C. P.; FILATRO A. C. O desenvolvimento profissional da docência na formação de professores face a utilização das tecnologias. Educ Temática Digital, v.10, n.2, p. 394-427, 2009.

PINTO, L. F.; ROCHA, C. M. F. Inovações na atenção Primária em Saúde: o uso de ferramentas de tecnologia de comunicação e informação para apoio à gestão local. Ciência \& Saúde Coletiva, v.21, n.5, p.1433-48, 2016. 
SANTOS, A . N.; MERCADO, L. P. L. Arquivamento e comunicação de imagens radiológicas na formação médica online. Revista Brasileira de Educação Médica, v.34, n.4, p.525-34, 2010.

SEELAND, U.; NAUMAN, A. T.; CORNELIS, A.; LUDWIG, S.; DUNKEL, M.; KARARIGAS, G; REGITZ-ZAGROSEK, V. eGender-from e-learning to e-research: a web-based interactive knowledge-sharing platform for sex- and gender-specific medical education. Biology of Sex Differences, v.7, n.1,2016.

SILVA, C. S.; SOUZA, M. B; FILHO, R. S. F.; MEDEIROS, L. M.; CRIADO P. R. E-learning program for medical students in dermatology. Clinics, v. 66, n.4, p.619-22, 2011.

SIMMONSEN, B. O; DAEHLIN, G. K.;JOHANSSON, I.; FARUP, P. Improvement of drug dose calculations by classroom teaching or e-learning: a randomised controlled trial in nurses. BMJ Open, v.4, 2014.

TAVEIRA-GOMES, T.; PRADO-COSTA, R.; SEVERO, M.; FERREIRA, M. A. Characterization of medical students recall of factual knowledge using learning objects and repeated testingin a novel e-learning system .BMC Medical Education, v.15, n.4, 2015.
TOBASE, L.; TOMAZINI, E. S.; TEODORO, S. V; PIZA, N. G; PERES, H. H. C. Ensino à distância na educação permanente em Urgência e Emergência. J. Health Inform, v.4, p.125-9, 2012.

YAO, K.; UEDO, N.; ISHIKAWA, H.; FILHO, E. C. C.; CARDONA, H. J.; PITTAYANON, R.; OLANO,K C.; YAO, F.; PARRA-BLANCO, A.; HO, S. H.; AVENDANO, A. G.; PISCOYA, A.; FEDOROV, E.; BIALEK, A. P.; MITRAKOV, A.; CARO, L.; GONEN, C.; DOLWANI, S.; FARCA, A.; CUARESMA, L. F.; BONILLA, J. J.; KASETSERMWIRIYA, W.; RAGUNATH, K.; KIM, S. E.; MARINI, M.; LI, H.; CIMMINO, D. G.; PISKORZ, M. M.; IACOPINI, F.; SO, J. B.; YAMAZAKI, K.; KIM, G. H.; ANG, T. L.; MILHOMEM-CARDOSO, D. M.; WALDBAUM, C. A.; CARVAJAL, W. A. P.; HAYWARD, C. M.; SINGH, R.; BANERJEE, R.; ANAGNOSTOPOULOS, G. K.; TAKAHASHI, Y. Development of an e-learning system for endoscopic diagnosis early gastric cancer: an international multicenter randomised controlled trial. Ebio Medicine, v.9, p.140-7, 2016.

XU, W.; JIANG, Q.; QIN, X.; FANG, G.; HU, Z. Elearning for grass-roots emergency public health personnel: preliminary lessons from a national program in China. BioScience Trends, v.10, n.3, p.235-9, 2016. 